\title{
Discretization error due to the identity operator in surface integral equations
}

\author{
Özgür Ergül ${ }^{\mathrm{a}, \mathrm{b}}$, Levent Gürel ${ }^{\mathrm{a}, \mathrm{b}, *}$ \\ a Department of Electrical and Electronics Engineering, Bilkent University, TR-06800, Ankara, Turkey \\ b Computational Electromagnetics Research Center (BiLCEM), Bilkent University, TR-06800, Ankara, Turkey
}

\section{A R T I C L E I N F O}

\section{Article history:}

Received 31 July 2008

Received in revised form 17 April 2009

Accepted 21 April 2009

Available online 3 May 2009

\section{Keywords:}

Surface integral equations

Low-order basis functions

First-kind integral equations

Second-kind integral equations

Identity operator

Accuracy analysis

\begin{abstract}
A B S T R A C T
We consider the accuracy of surface integral equations for the solution of scattering and radiation problems in electromagnetics. In numerical solutions, second-kind integral equations involving welltested identity operators are preferable for efficiency, because they produce diagonally-dominant matrix equations that can be solved easily with iterative methods. However, the existence of the well-tested identity operators leads to inaccurate results, especially when the equations are discretized with low-order basis functions, such as the Rao-Wilton-Glisson functions. By performing a computational experiment based on the nonradiating property of the tangential incident fields on arbitrary surfaces, we show that the discretization error of the identity operator is a major error source that contaminates the accuracy of the second-kind integral equations significantly.
\end{abstract}

(C) 2009 Elsevier B.V. All rights reserved.

\section{Glossary}

CFIE: Combined-field integral equation

CTF: Combined tangential formulation

EFIE: $\quad$ Electric-field integral equation

JMCFIE: Electric and magnetic current combined-field integral equation

MFIE: Magnetic-field integral equation

MNMF: Modified normal Müller formulation

PEC: $\quad$ Perfect electric conductor

RMS: $\quad$ Root-mean square

RWG: Rao-Wilton-Glisson (functions)

\section{Introduction}

Surface integral equations are commonly used for the solution of scattering and radiation problems in electromagnetics [1]. Equivalent currents are defined on the surface of the object, and the boundary conditions are used to derive a set of equations to solve the equivalent currents and to calculate the scattered or radiated electromagnetic fields. For a perfect electric conductor (PEC), the electric-field integral equation (EFIE) and the magnetic-field integral equation (MFIE) can be derived by testing the boundary conditions for the tangential electric and magnetic fields, respectively,

\footnotetext{
* Corresponding author at: Department of Electrical and Electronics Engineering, Bilkent University, TR-06800, Ankara, Turkey. Tel.: +90 312290 5750; fax: +90 312 2905755.

E-mail address: lgurel@bilkent.edu.tr (L. Gürel).
}

on the surface of the object. For closed surfaces, EFIE and MFIE can be combined to obtain a combined-field integral equation (CFIE), which is free of the internal-resonance problem [2]. For dielectric objects with homogeneous material properties, various formulations can be obtained by testing the boundary conditions on the two sides (i.e., inner and outer sides) of the surfaces and combining the equations properly. Among those formulations, the combined tangential formulation (CTF) [3], the modified normal Müller formulation (MNMF) [4], and the electric and magnetic current combined-field integral equation (JMCFIE) [5] are preferable, due to their stable solutions. CTF and MNMF are improved versions of the well-known Poggio-Miller-Chang-Harrington-Wu-Tsai (PMCHWT) [1] and normal Müller [6] formulations, respectively.

Surface formulations can be categorized into three groups, i.e., tangential, normal, and mixed formulations, depending on the testing scheme used for the boundary conditions. In the tangential formulations, such as EFIE and CTF, boundary conditions are tested directly by sampling the tangential components of the electric and magnetic fields on the surface. However, in the normal formulations, such as MFIE and MNMF, fields are tested after they are projected onto the surface by using the outward normal vector. Finally, the mixed formulations, such as CFIE and JMCFIE, involve both types of the testing schemes. Using a Galerkin method in the discretization of the surface formulations, i.e., using the same set of functions to expand the current densities and to test the boundary conditions, the normal and mixed formulations contain well-tested identity operators. Then, these formulations produce well-conditioned matrix equations since the well-tested identity operators lead to diagonally-dominant matrices. We note that the existence of the identity operator alone is not sufficient for a well- 
conditioned matrix equation. Nevertheless, a small perturbation of the identity operator with contributions from the other operators leads to a well-conditioned system. On the other hand, the tangential formulations do not contain well-tested identity operators, and their discretizations may lead to ill-conditioned matrix equations.

For all of tangential, normal, and mixed formulations considered in this paper, boundary conditions are always tested tangentially on the surface either directly or by a projection using the outward normal vector. In other words, only the tangential components of the electric and magnetic fields are tested to derive the integral equations. We note that there are also other integralequation formulations, which involve normal components of the electric and magnetic fields on surfaces. Those formulations are not considered in this work and they should not be confused with the normal formulations presented in this paper.

Depending on the use of the identity operator, the normal and mixed formulations, i.e., MFIE, MNMF, CFIE, and JMCFIE, are second-kind integral equations, while EFIE is a first-kind integral equation. CTF is somewhat in between, because it contains an identity operator, even though the identity operator is not well tested. Nevertheless, CTF is closer to the category of first-kind integral equations since it does not lead to diagonally-dominant and well-conditioned matrices. For the efficiency of the solutions, second-kind integral equations are preferable, especially when problems involve large objects discretized with large numbers of unknowns [7].

In addition to the conditioning of the matrix equations, the identity operator plays a key role in the accuracy of the solutions. Recent investigations on PEC objects show that the scattered fields obtained with MFIE and CFIE are significantly inaccurate in comparison to those obtained with EFIE [8,9]. Inaccuracy of MFIE and CFIE becomes obvious, especially when they are discretized with low-order basis functions, such as the Rao-Wilton-Glisson (RWG) functions [10] defined on planar triangles. It was also shown that the accuracy of MFIE and CFIE can be improved to the levels of EFIE by employing more appropriate discretizations, especially with higher-order basis functions, instead of the RWG functions [11-17]. Similar observations have been made for the solution of dielectric objects [3]. Finally, recent studies show that the regularization of the identity operator improves the accuracy of MFIE [9, 18].

In this paper, we show that the identity operator is truly a major error source in surface formulations that are discretized with low-order basis functions. We demonstrate the inaccuracy problem by setting up a computational experiment based on the nonradiating property of the tangential incident fields on an arbitrary surface. Incident fields, which are analytically defined in a continuous space, are discretized and expanded in a series of RWG functions by using two methods, i.e., an expansion employing the identity operator and an expansion employing directly-tested integrodifferential operators. We show that the first method involving well-tested identity operators is significantly inaccurate compared to the second method. The two discretization methods are related to the solutions of electromagnetics problems with second-kind and first-kind integral equations, respectively, in which the total currents are expanded in a series of basis functions.

\section{Surface integral equations}

For the surface formulations of scattering and radiation problems, three different operators can be defined as

$\mathcal{T}\{\boldsymbol{X}\}(\boldsymbol{r})=i k \int_{S} d \boldsymbol{r}^{\prime}\left[\boldsymbol{X}\left(\boldsymbol{r}^{\prime}\right)+\frac{1}{k^{2}} \nabla^{\prime} \cdot \boldsymbol{X}\left(\boldsymbol{r}^{\prime}\right) \nabla\right] g\left(\boldsymbol{r}, \boldsymbol{r}^{\prime}\right)$,
$\mathcal{K}\{\boldsymbol{X}\}(\boldsymbol{r})=\int_{S} d \boldsymbol{r}^{\prime} \boldsymbol{X}\left(\boldsymbol{r}^{\prime}\right) \times \nabla^{\prime} g\left(\boldsymbol{r}, \boldsymbol{r}^{\prime}\right)$,

$\mathcal{I}\{\boldsymbol{X}\}(\boldsymbol{r})=\boldsymbol{X}(\boldsymbol{r})$,

where $S$ is the closed surface of a three-dimensional object with an arbitrary shape. In (1)-(3), $\boldsymbol{X}$ is either the equivalent electric current $(\boldsymbol{J})$ or the equivalent magnetic current $(\boldsymbol{M})$ on the surface, $k=\omega \sqrt{\mu \epsilon}$ is the wavenumber, and $g\left(\boldsymbol{r}, \boldsymbol{r}^{\prime}\right)$ denotes the homogeneous-space Green's function defined as

$g\left(\boldsymbol{r}, \boldsymbol{r}^{\prime}\right)=\frac{\exp (i k R)}{4 \pi R} \quad\left(R=\left|\boldsymbol{r}-\boldsymbol{r}^{\prime}\right|\right)$

in phasor notation with the $e^{-i \omega t}$ convention. The operator $\mathcal{K}$ is commonly separated into principle-value and limit parts as

$\mathcal{K}\{\boldsymbol{X}\}(\boldsymbol{r})=\mathcal{K}_{P V}\{\boldsymbol{X}\}(\boldsymbol{r})-\frac{\Omega_{i}(\boldsymbol{r})}{4 \pi} \mathcal{I}^{\times n}\{\boldsymbol{X}\}(\boldsymbol{r})$,

where $0 \leqslant \Omega_{i}(\boldsymbol{r}) \leqslant 4 \pi$ is the internal solid angle, which is nonzero when the observation point $\boldsymbol{r}$ is on the surface [19]. In addition, $\mathcal{I}^{\times n}\{\boldsymbol{X}\}(\boldsymbol{r})=\hat{\boldsymbol{n}} \times \boldsymbol{X}(\boldsymbol{r})$, where $\hat{\boldsymbol{n}}$ is the outward normal vector.

Using the equivalent surface currents, i.e.,

$\boldsymbol{J}(\boldsymbol{r})=\mathcal{I}^{\times n}\{\boldsymbol{H}\}(\boldsymbol{r})=\hat{\boldsymbol{n}} \times \boldsymbol{H}(\boldsymbol{r})$,

$\boldsymbol{M}(\boldsymbol{r})=-\mathcal{I}^{\times n}\{\boldsymbol{E}\}(\boldsymbol{r})=-\hat{\boldsymbol{n}} \times \boldsymbol{E}(\boldsymbol{r})$,

scattered (or radiated) electric and magnetic fields can be calculated as

$\boldsymbol{E}^{s c a}(\boldsymbol{r})=\eta \mathcal{T}\{\boldsymbol{J}\}(\boldsymbol{r})-\mathcal{K}_{P V}\{\boldsymbol{M}\}(\boldsymbol{r})+\frac{\Omega_{i}(\boldsymbol{r})}{4 \pi} \mathcal{I}^{\times n}\{\boldsymbol{M}\}(\boldsymbol{r})$,
$\boldsymbol{H}^{s c a}(\boldsymbol{r})=\frac{1}{\eta} \mathcal{T}\{\boldsymbol{M}\}(\boldsymbol{r})+\mathcal{K}_{P V}\{\boldsymbol{J}\}(\boldsymbol{r})-\frac{\Omega_{i}(\boldsymbol{r})}{4 \pi} \mathcal{I}^{\times n}\{\boldsymbol{J}\}(\boldsymbol{r})$,

where $\eta=\sqrt{\mu / \epsilon}$ is the wave impedance. For PEC objects, the tangential component of the total electric field vanishes on the surface $(\boldsymbol{M}=0)$. Then, using the boundary conditions for the tangential electric and magnetic fields, we obtain EFIE and MFIE as

$\hat{\boldsymbol{t}} \cdot \eta \mathcal{T}\{\boldsymbol{J}\}(\boldsymbol{r})=-\hat{\boldsymbol{t}} \cdot \boldsymbol{E}^{i n c}(\boldsymbol{r})$

and

$\hat{\boldsymbol{n}} \times \mathcal{K}_{P V}\{\boldsymbol{J}\}(\boldsymbol{r})-\frac{\Omega_{0}(\boldsymbol{r})}{4 \pi} \mathcal{I}\{\boldsymbol{J}\}(\boldsymbol{r})=-\hat{\boldsymbol{n}} \times \boldsymbol{H}^{i n c}(\boldsymbol{r})$,

respectively, where $\Omega_{0}(\boldsymbol{r})$ is the external solid angle at the observation point $\boldsymbol{r} \in S, \hat{\boldsymbol{t}}$ is any tangential vector on the surface, and $\boldsymbol{E}^{\text {inc }}(\boldsymbol{r})$ and $\boldsymbol{H}^{\text {inc }}(\boldsymbol{r})$ are the incident electric and magnetic fields produced by the external sources. We note that the boundary conditions for the electric and magnetic fields are tested via the tangential $(\hat{\boldsymbol{t}})$ and normal $(\hat{\boldsymbol{n}})$ unit vectors, respectively, to obtain stable solutions using a Galerkin scheme. Otherwise, the resulting matrix equations are extremely ill-conditioned and cannot be solved iteratively. Consequently, EFIE and MFIE are tangential and normal formulations, respectively. For closed surfaces, EFIE and MFIE can be combined linearly to obtain a mixed formulation, i.e., CFIE, which is free of the internal-resonance problem. Both MFIE and CFIE are second-kind integral equations, which produce betterconditioned matrix equations, compared to those obtained with the first-kind EFIE [20].

For dielectric objects, boundary conditions are used on both sides of the surfaces to derive two sets of equations for the inner and outer problems. Then, the inner and outer equations can be combined in different ways to obtain various formulations for the solution of the equivalent electric and magnetic currents on the surfaces. Among infinitely many possibilities, CTF, MNMF, and JMCFIE are natural extensions of EFIE, MFIE, and CFIE, respectively, for dielectric objects [3-5]. All three formulations are free of the internal-resonance problem. Similar to MFIE and CFIE, MNMF and 
JMCFIE are second-kind integral equations, and they produce wellconditioned matrix equations. Dielectric formulations are derived in Appendix A.

In surface formulations of closed objects, equivalent surface currents can be decomposed as

$\boldsymbol{J}(\boldsymbol{r})=\hat{\boldsymbol{n}} \times \boldsymbol{H}(\boldsymbol{r})=\hat{\boldsymbol{n}} \times \boldsymbol{H}^{i n c}(\boldsymbol{r})+\hat{\boldsymbol{n}} \times \boldsymbol{H}^{s c a}(\boldsymbol{r})$,

$\boldsymbol{M}(\boldsymbol{r})=-\hat{\boldsymbol{n}} \times \boldsymbol{E}(\boldsymbol{r})=-\hat{\boldsymbol{n}} \times \boldsymbol{E}^{i n c}(\boldsymbol{r})-\hat{\boldsymbol{n}} \times \boldsymbol{E}^{s c a}(\boldsymbol{r})$,

where $\left\{\boldsymbol{J}^{\text {inc }}(\boldsymbol{r}), \boldsymbol{M}^{\text {inc }}(\boldsymbol{r})\right\}=\left\{\hat{\boldsymbol{n}} \times \boldsymbol{H}^{\text {inc }}(\boldsymbol{r}),-\hat{\boldsymbol{n}} \times \boldsymbol{E}^{i n c}(\boldsymbol{r})\right\}$ do not radiate outside the surface $[21,22]$, i.e.,

$\eta \mathcal{T}\left\{\boldsymbol{J}^{i n c}\right\}(\boldsymbol{r})-\mathcal{K}_{P V}\left\{\boldsymbol{M}^{i n c}\right\}(\boldsymbol{r})+\frac{\Omega_{i}(\boldsymbol{r})}{4 \pi} \mathcal{I}^{\times n}\left\{\boldsymbol{M}^{i n c}\right\}(\boldsymbol{r})=0$,

$\frac{1}{\eta} \mathcal{T}\left\{\boldsymbol{M}^{i n c}\right\}(\boldsymbol{r})+\mathcal{K}_{P V}\left\{\boldsymbol{J}^{i n c}\right\}(\boldsymbol{r})-\frac{\Omega_{i}(\boldsymbol{r})}{4 \pi} \mathcal{I}^{\times n}\left\{\boldsymbol{J}^{i n c}\right\}(\boldsymbol{r})=0$.

For PEC surfaces,

$\boldsymbol{M}^{i n c}(\boldsymbol{r})=-\hat{\boldsymbol{n}} \times \boldsymbol{E}^{i n c}(\boldsymbol{r})=\hat{\boldsymbol{n}} \times \boldsymbol{E}^{s c a}(\boldsymbol{r})$.

Rearranging the terms in (14)-(15), one can obtain

$$
\begin{aligned}
& {\left[\begin{array}{cc}
\mathcal{T} & -\eta^{-1} \mathcal{K}_{P V} \\
\eta \mathcal{K}_{P V} & \mathcal{T}
\end{array}\right] \cdot\left[\begin{array}{c}
\hat{\boldsymbol{n}} \times \boldsymbol{H}^{i n c} \\
-\hat{\boldsymbol{n}} \times \boldsymbol{E}^{i n c}
\end{array}\right](\boldsymbol{r})} \\
& =-\frac{\Omega_{i}(\boldsymbol{r})}{4 \pi}\left[\begin{array}{c}
\eta^{-1} \boldsymbol{E}^{i n c}(\boldsymbol{r}) \\
\eta \boldsymbol{H}^{i n c}(\boldsymbol{r})
\end{array}\right] .
\end{aligned}
$$

The decomposition in (12)-(13) is useful for accurate solutions of low-contrast dielectric problems [22]. In this study, we use the same decomposition and the nonradiating property of $\left\{\boldsymbol{J}^{i n c}(\boldsymbol{r})\right.$, $\left.\boldsymbol{M}^{i n c}(\boldsymbol{r})\right\}$ to determine the error caused by the discretization of the integro-differential and identity operators.

\section{Discretization of the surface integral equations}

For numerical solutions of surface integral equations, equivalent currents are expanded in a series of basis functions $\boldsymbol{b}_{n}(\boldsymbol{r})$, i.e.,

$$
\begin{aligned}
& \boldsymbol{J}(\boldsymbol{r})=\sum_{n=1}^{N} x_{n} \boldsymbol{b}_{n}(\boldsymbol{r}), \\
& \boldsymbol{M}(\boldsymbol{r})=\sum_{n=1}^{N} y_{n} \boldsymbol{b}_{n}(\boldsymbol{r}) .
\end{aligned}
$$

Testing the integral equations using a set of functions $\boldsymbol{t}_{m}(\boldsymbol{r})$, matrix equations are constructed and solved to calculate the unknown coefficients $x_{n}$ and/or $y_{n}$. We consider a Galerkin scheme and use the same set of RWG functions as the basis and testing functions. For example, EFIE and MFIE in (10) and (11) can be discretized as

$\sum_{n=1}^{N} x_{n} \eta \int_{S_{m}} d \boldsymbol{r} \boldsymbol{t}_{m}(\boldsymbol{r}) \cdot \mathcal{T}\left\{\boldsymbol{b}_{n}\right\}(\boldsymbol{r})=-\int_{S_{m}} d \boldsymbol{r} \boldsymbol{t}_{m}(\boldsymbol{r}) \cdot \boldsymbol{E}^{i n c}(\boldsymbol{r})$

and

$$
\begin{aligned}
\sum_{n=1}^{N} & x_{n} \int_{S_{m}} d \boldsymbol{r} \boldsymbol{t}_{m}(\boldsymbol{r}) \cdot \hat{\boldsymbol{n}} \times \mathcal{K}_{P V}\left\{\boldsymbol{b}_{n}\right\}(\boldsymbol{r}) \\
& -\sum_{n=1}^{N} x_{n} \frac{1}{4 \pi} \int_{S_{m}} d \boldsymbol{r} \Omega_{o}(\boldsymbol{r}) \boldsymbol{t}_{m}(\boldsymbol{r}) \cdot \mathcal{I}\left\{\boldsymbol{b}_{n}\right\}(\boldsymbol{r}) \\
& =-\int_{S_{m}} d \boldsymbol{r} \boldsymbol{t}_{m}(\boldsymbol{r}) \cdot \hat{\boldsymbol{n}} \times \boldsymbol{H}^{i n c}(\boldsymbol{r}),
\end{aligned}
$$

respectively, where $S_{m}$ is the spatial support of the $m$ th RWG function for $m=1,2, \ldots, N$.
Using a Galerkin scheme, the $\mathcal{T}$ operator is well-tested when it is directly tested with $\boldsymbol{t}_{m}(\boldsymbol{r})$ [3], and EFIE involves a well-tested $\mathcal{T}$ operator, i.e.,

$$
\begin{aligned}
T_{m n}= & \int_{S_{m}} d \boldsymbol{r} \boldsymbol{t}_{m}(\boldsymbol{r}) \cdot \mathcal{T}\left\{\boldsymbol{b}_{n}\right\}(\boldsymbol{r}) \\
= & i k \int_{S_{m}} d \boldsymbol{r} \boldsymbol{t}_{m}(\boldsymbol{r}) \cdot \int_{S_{n}} d \boldsymbol{r}^{\prime} \boldsymbol{b}_{n}(\boldsymbol{r}) g\left(\boldsymbol{r}, \boldsymbol{r}^{\prime}\right) \\
& -\frac{i}{k} \int_{S_{m}} d \boldsymbol{r} \boldsymbol{t}_{m}(\boldsymbol{r}) \cdot \int_{S_{n}} d \boldsymbol{r}^{\prime} \nabla^{\prime} \cdot \boldsymbol{b}_{n}(\boldsymbol{r}) \nabla g\left(\boldsymbol{r}, \boldsymbol{r}^{\prime}\right) .
\end{aligned}
$$

Using the divergence-conforming RWG functions, the hyper-singularity in the second term of (22) is eliminated by moving the differential operator onto the testing function [10], i.e.,

$$
\begin{aligned}
T_{m n}= & i k \int_{S_{m}} d \boldsymbol{r} \boldsymbol{t}_{m}(\boldsymbol{r}) \cdot \int_{S_{n}} d \boldsymbol{r}^{\prime} \boldsymbol{b}_{n}(\boldsymbol{r}) g\left(\boldsymbol{r}, \boldsymbol{r}^{\prime}\right) \\
& +\frac{i}{k} \int_{S_{m}} d \boldsymbol{r} \nabla \cdot \boldsymbol{t}_{m}(\boldsymbol{r}) \int_{S_{n}} d \boldsymbol{r}^{\prime} \nabla^{\prime} \cdot \boldsymbol{b}_{n}(\boldsymbol{r}) g\left(\boldsymbol{r}, \boldsymbol{r}^{\prime}\right) .
\end{aligned}
$$

As opposed to the $\mathcal{T}$ operator, the $\mathcal{K}$ operator is well-tested with $\hat{\boldsymbol{n}} \times \boldsymbol{t}_{m}(\boldsymbol{r})$ [3]. ${ }^{1}$ MFIE involves well-tested $\mathcal{K}$ and identity operators, i.e.,

$$
\begin{aligned}
K_{m n}^{\times n}= & \int_{S_{m}} d \boldsymbol{r} \boldsymbol{t}_{m}(\boldsymbol{r}) \cdot \hat{\boldsymbol{n}} \times \mathcal{K}_{P V}\left\{\boldsymbol{b}_{n}\right\}(\boldsymbol{r}) \\
& -\frac{1}{4 \pi} \int_{S_{m}} d \boldsymbol{r} \Omega_{o}(\boldsymbol{r}) \boldsymbol{t}_{m}(\boldsymbol{r}) \cdot \mathcal{I}\left\{\boldsymbol{b}_{n}\right\}(\boldsymbol{r}) \\
= & \int_{S_{m}} d \boldsymbol{r} \boldsymbol{t}_{m}(\boldsymbol{r}) \cdot \hat{\boldsymbol{n}} \times \int_{S_{n}, P V} d \boldsymbol{r}^{\prime} \boldsymbol{b}\left(\boldsymbol{r}^{\prime}\right) \times \nabla^{\prime} g\left(\boldsymbol{r}, \boldsymbol{r}^{\prime}\right) \\
& -\frac{1}{2} \int_{S_{m}} d \boldsymbol{r} \boldsymbol{t}_{m}(\boldsymbol{r}) \cdot \boldsymbol{b}_{n}(\boldsymbol{r}) \\
= & K_{m n, P V}^{\times n}-\frac{1}{2} I_{m n}
\end{aligned}
$$

since $\Omega_{0}(\boldsymbol{r})=1 / 2$ on planar surfaces. The value of $I_{m n}$ in (24) is nonzero for overlapping testing and basis functions, and it typically has a large value for $m=n$. This is why the matrix equations obtained with MFIE and CFIE are diagonally dominant and easy to solve iteratively.

CTF, which is a tangential formulation for dielectric objects, involves well-tested $\mathcal{T}$ operators, similar to EFIE. In addition, CTF contains weakly-tested $\mathcal{K}$ and identity operators, i.e.,

$$
\begin{aligned}
& K_{m n, P V}=\int_{S_{m}} d \boldsymbol{r} \boldsymbol{t}_{m}(\boldsymbol{r}) \cdot \mathcal{K}_{P V}\left\{\boldsymbol{b}_{n}\right\}(\boldsymbol{r}), \\
& I_{m n}^{\times n}=\int_{S_{m}} d \boldsymbol{r} \boldsymbol{t}_{m}(\boldsymbol{r}) \cdot \hat{\boldsymbol{n}} \times \boldsymbol{b}_{n}(\boldsymbol{r}) .
\end{aligned}
$$

We note that $I_{m n}^{\times n} \approx 0$ for $m=n$, and CTF is "practically" a first-kind integral equation, even though it involves the identity operator. Similar to MFIE, MNMF contains well-tested $\mathcal{K}$ and identity operators. Besides, this formulation involves weakly-tested $\mathcal{T}$ operators, i.e.,

$T_{m n}^{\times n}=\int_{S_{m}} d \boldsymbol{r} \boldsymbol{t}_{m}(\boldsymbol{r}) \cdot \hat{\boldsymbol{n}} \times \mathcal{T}\left\{\boldsymbol{b}_{n}\right\}(\boldsymbol{r})$.

\footnotetext{
1 Testing with $\hat{\boldsymbol{n}} \times \boldsymbol{t}_{m}(\boldsymbol{r})$ is equivalent to testing with $\boldsymbol{t}_{m}(\boldsymbol{r})$ after a projection onto the surface by using $\hat{\boldsymbol{n}}$, i.e., $\boldsymbol{t}_{m}(\boldsymbol{r}) \cdot \hat{\boldsymbol{n}} \times$.
} 
In general, conditioning and accuracy properties of the tangential formulations, i.e., EFIE and CTF, are determined by the well-tested $\mathcal{T}$ operator. Using a Galerkin scheme and divergence-conforming RWG functions, the directly-tested $\mathcal{T}$ operator has a weaklysingular kernel [23]. This leads to ill-conditioned matrix equations, but very accurate results, provided that the condition number of the matrix is not too large to deteriorate the accuracy of the solutions. On the other hand, the normal and mixed formulations, namely, MFIE, MNMF, CFIE, and JMCFIE, are dominated by the welltested identity operators. This is preferable in terms of efficiency since diagonally-dominant matrix equations are well-conditioned and easy to solve iteratively. However, as demonstrated in the next section, discretization of the identity operator involves a large error, which significantly contaminates the accuracy of the results.

\section{Discretization error due to the identity operator}

Using the RWG functions on planar triangles, discretization of the well-tested identity operator is trivial. The integral

$$
I_{m n}=\int_{S_{m}} d \boldsymbol{r} \boldsymbol{t}_{m}(\boldsymbol{r}) \cdot \boldsymbol{b}_{n}(\boldsymbol{r})
$$

does not contain any singularity and it can be evaluated accurately (even exactly, if desired) by using a low-order Gaussian quadrature rule. On the other hand, the identity operator behaves like an operator with a highly-singular kernel $[9,23]$. This alternative interpretation can be understood when (28) is rewritten as a double integral over the testing and basis functions as

$I_{m n}=\int_{S_{m}} d \boldsymbol{r} \boldsymbol{t}_{m}(\boldsymbol{r}) \cdot \int_{S_{n}} d \boldsymbol{r}^{\prime} \delta\left(\boldsymbol{r}, \boldsymbol{r}^{\prime}\right) \boldsymbol{b}_{n}(\boldsymbol{r})$,

where $\delta\left(\boldsymbol{r}, \boldsymbol{r}^{\prime}\right)$ is a Dirac delta function as the kernel of the operator. Therefore, the discretization of the identity operator may cause an unexpectedly large error, even though the discretization itself, i.e., the expression in (28), involves very small or no error.

To present the excessive error due to the discretization of the identity operator, we consider the expansion of nonradiating currents $\left\{\boldsymbol{J}^{\text {inc }}(\boldsymbol{r}), \boldsymbol{M}^{\text {inc }}(\boldsymbol{r})\right\}$ in a series of RWG functions, i.e.,

$$
\begin{aligned}
& \boldsymbol{J}^{i n c}(\boldsymbol{r})=\hat{\boldsymbol{n}} \times \boldsymbol{H}^{i n c}(\boldsymbol{r})=\sum_{n=1}^{N} x_{n}^{i n c} \boldsymbol{b}_{n}(\boldsymbol{r}), \\
& \boldsymbol{M}^{i n c}(\boldsymbol{r})=-\hat{\boldsymbol{n}} \times \boldsymbol{E}^{i n c}(\boldsymbol{r})=\sum_{n=1}^{N} y_{n}^{i n c} \boldsymbol{b}_{n}(\boldsymbol{r}) .
\end{aligned}
$$

For an arbitrary surface, unknown coefficients $x_{n}^{\text {inc }}$ and $y_{n}^{\text {inc }}$ can be calculated by using two different methods:

(i) The equality in (17) is discretized and solved. Similar to tangential formulations, we project and test the equation on the surface via a set of testing functions $\boldsymbol{t}_{m}(\boldsymbol{r})$ to obtain a $2 \mathrm{~N} \times 2 \mathrm{~N}$ matrix equation, i.e.,

$\left[\begin{array}{cc}\overline{\boldsymbol{T}} & -\eta^{-1} \overline{\boldsymbol{K}}_{P V} \\ \eta \overline{\boldsymbol{K}}_{P V} & \overline{\boldsymbol{T}}\end{array}\right] \cdot\left[\begin{array}{l}\boldsymbol{x}^{i n c} \\ \boldsymbol{y}^{i n c}\end{array}\right]=-\frac{1}{2}\left[\begin{array}{c}\eta^{-1} \boldsymbol{e}^{i n c} \\ \eta \boldsymbol{h}^{i n c}\end{array}\right]$,

where

$$
\begin{aligned}
& \overline{\boldsymbol{T}}[m, n]=T_{m n}=\int_{S_{m}} d \boldsymbol{r} \boldsymbol{t}_{m}(\boldsymbol{r}) \cdot \mathcal{T}\left\{\boldsymbol{b}_{n}\right\}(\boldsymbol{r}), \\
& \overline{\boldsymbol{K}}_{P V}[m, n]=K_{m n, P V}=\int_{S_{m}} d \boldsymbol{r} \boldsymbol{t}_{m}(\boldsymbol{r}) \cdot \mathcal{K}_{P V}\left\{\boldsymbol{b}_{n}\right\}(\boldsymbol{r}), \\
& \boldsymbol{h}^{i n c}[m]=\int_{S_{m}} d \boldsymbol{r} \boldsymbol{t}_{m}(\boldsymbol{r}) \cdot \boldsymbol{H}^{i n c}(\boldsymbol{r}),
\end{aligned}
$$

$$
\begin{aligned}
& \boldsymbol{e}^{i n c}[m]=\int_{S_{m}} d \boldsymbol{r} \boldsymbol{t}_{m}(\boldsymbol{r}) \cdot \boldsymbol{E}^{i n c}(\boldsymbol{r}), \\
& \boldsymbol{x}^{i n c}[m]=x_{m}^{i n c}, \quad \boldsymbol{y}^{i n c}[m]=y_{m}^{i n c}
\end{aligned}
$$

for $m, n=1,2, \ldots, N$. We note that this method does not involve any (well-tested) identity operator.

(ii) We consider an identity equation in the form of

$$
\left[\begin{array}{cc}
\mathcal{I} & 0 \\
0 & \mathcal{I}
\end{array}\right] \cdot\left[\begin{array}{c}
\hat{\boldsymbol{n}} \times \boldsymbol{H}^{i n c} \\
-\hat{\boldsymbol{n}} \times \boldsymbol{E}^{i n c}
\end{array}\right]=\left[\begin{array}{c}
\hat{\boldsymbol{n}} \times \boldsymbol{H}^{i n c} \\
-\hat{\boldsymbol{n}} \times \boldsymbol{E}^{i n c}
\end{array}\right],
$$

where the nonradiating currents on the left-hand side are expanded in a series of basis functions. Discretization of (38) leads to a $2 N \times 2 N$ matrix equation, i.e.,

$$
\left[\begin{array}{cc}
\overline{\boldsymbol{I}} & 0 \\
0 & \overline{\boldsymbol{I}}
\end{array}\right] \cdot\left[\begin{array}{c}
\boldsymbol{x}^{i n c} \\
\boldsymbol{y}^{i n c}
\end{array}\right]=\left[\begin{array}{c}
\boldsymbol{h}_{\times n}^{i n c} \\
-\boldsymbol{e}_{\times n}^{i n c}
\end{array}\right],
$$

where

$$
\begin{aligned}
& \overline{\mathbf{I}}[m, n]=I_{m n}=\int_{S_{m}} d \boldsymbol{r} \boldsymbol{t}_{m}(\boldsymbol{r}) \cdot \boldsymbol{b}_{n}(\boldsymbol{r}), \\
& \boldsymbol{h}_{\times n}^{i n c}[m]=\int_{S_{m}} d \boldsymbol{r} \boldsymbol{t}_{m}(\boldsymbol{r}) \cdot \hat{\boldsymbol{n}} \times \boldsymbol{H}^{i n c}(\boldsymbol{r}), \\
& \boldsymbol{e}_{\times n}^{i n c}[m]=\int_{S_{m}} d \boldsymbol{r} \boldsymbol{t}_{m}(\boldsymbol{r}) \cdot \hat{\boldsymbol{n}} \times \boldsymbol{E}^{i n c}(\boldsymbol{r})
\end{aligned}
$$

for $m, n=1,2, \ldots, N$. Clearly, this second method is remarkably simpler than the first one, as it does not involve the discretization of any integro-differential operator.

We perform experiments on two different geometries: a sphere with a radius of $0.5 \lambda$ and a cube with edges of $0.5 \lambda$. Both objects are illuminated by a plane wave propagating in the $-x$-direction with the electric field polarized in the $y$-direction. Amplitude of the plane wave is unity. Nonradiating currents $\left\{\boldsymbol{J}^{\text {inc }}(\boldsymbol{r}), \boldsymbol{M}^{\text {inc }}(\boldsymbol{r})\right\}$ are expanded in a series of RWG functions on the objects using the two methods described above, i.e., using directly-tested $\mathcal{K}$ and $\mathcal{T}$ operators and using the identity operator. Expansion coefficients are calculated and used to compute the radiated fields in the far zone. We define the far-zone electric field on the $x-y$ plane as

$$
E^{\infty}[p]=\lim _{r \rightarrow \infty}\left\{r \sqrt{\left|E_{\theta}\left(r, \theta, \phi_{p}\right)\right|^{2}+\left|E_{\phi}\left(r, \theta, \phi_{p}\right)\right|^{2}}\right\},
$$

where $E_{\theta}$ and $E_{\phi}$ are the $\theta$ and $\phi$ components of the radiated electric field, $\theta=\pi / 2$, and $\phi_{p}=(p-1) \pi / 180$ for $p=1,2, \ldots, 360$. Then, the root-mean square (RMS) of the far-zone field is calculated as

$\operatorname{RMS}\left\{\boldsymbol{E}^{\infty}\right\}=\sqrt{\frac{1}{360} \sum_{p=1}^{360}\left(E^{\infty}[p]\right)^{2}}$.

Since the expanded currents $\left\{\boldsymbol{J}^{\text {inc }}(\boldsymbol{r}), \boldsymbol{M}^{\text {inc }}(\boldsymbol{r})\right\}$ should not radiate, the RMS value in (44) directly corresponds to the error in the calculations. Using the RWG functions defined on planar triangles, we compute the far-zone fields analytically; hence, the error is only due to the discretization of the currents.

Figs. 1(a) and 1(b) present the RMS of the far-zone field for the sphere and the cube problems, respectively, as a function of the mesh size. In both cases, we observe that the RMS value decreases as the triangulation becomes finer. However, there exists a significant discrepancy between the results obtained with the two discretization methods. Given a mesh size, the RMS value is consistently smaller with the first method using the integro-differential operators $\mathcal{K}$ and $\mathcal{T}$ compared to the second method using the 


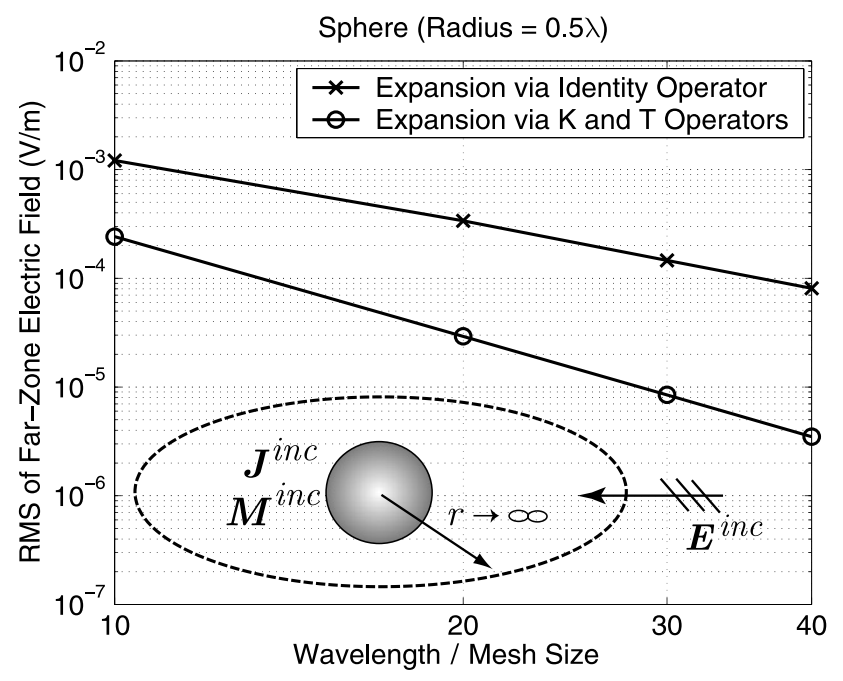

(a)

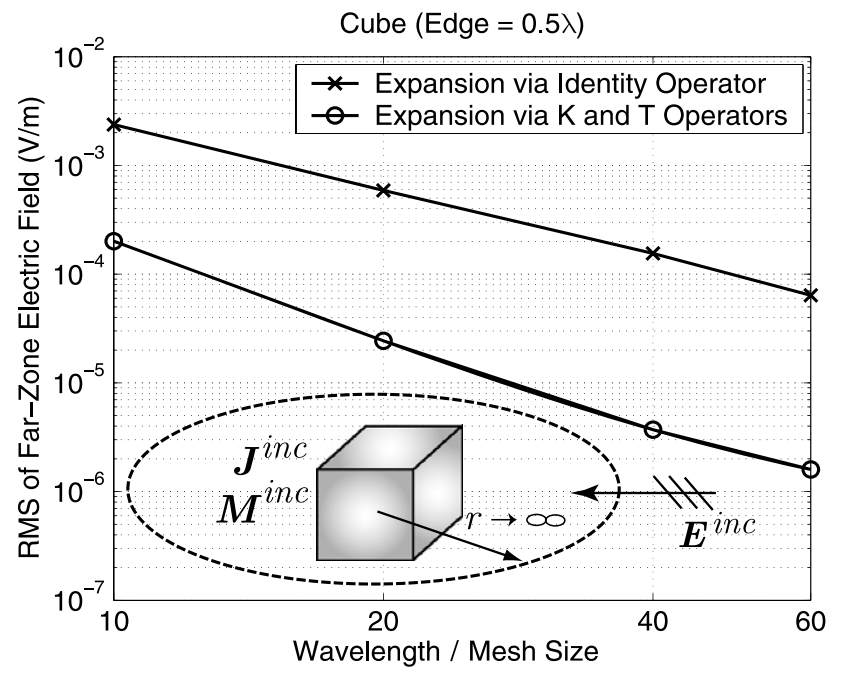

(b)

Fig. 1. RMS of the far-zone electric field due to nonradiating currents $\left\{\boldsymbol{J}^{\text {inc }}(\boldsymbol{r}), \boldsymbol{M}^{\text {inc }}(\boldsymbol{r})\right\}$ expanded on the surface of (a) a sphere with a radius of $0.5 \lambda$ and (b) a cube with $0.5 \lambda$ edges. Both surfaces are illuminated by a plane wave with unit amplitude propagating in free space.

well-tested identity operator. In other words, the discretization of the well-tested identity operator generates larger error compared to the discretization of the directly-tested $\mathcal{K}$ and $\mathcal{T}$ operators, even when we use the same set of RWG functions.

\section{Accuracy of surface formulations}

Normal and mixed surface formulations involving well-tested identity operators, such as MFIE, CFIE, MNMF, and JMCFIE, are noticeably and consistently more inaccurate than the tangential formulations, such as EFIE and CTF. This is mostly due to the excessive inaccuracy caused by the low-order discretization of the identity operator, as demonstrated in Section 4. As an example, Fig. 2 presents the results of a scattering problem involving a PEC sphere of radius $0.5 \lambda$ located in free space. The sphere is illuminated by a plane wave propagating in the $-x$-direction with the electric field polarized in the $y$-direction having an amplitude of unity. The problem is discretized with various mesh sizes and solved with EFIE and CFIE, which is obtained by the combination of EFIE and MFIE as $0.2 \times$ EFIE $+0.8 \times$ MFIE. Solving for the total electric current, the scattered electric field is calculated in the far zone on the $x-y$ plane at $\boldsymbol{r}=(r, \theta, \phi)=\left(\infty, \pi / 2, \phi_{p}\right)$, where

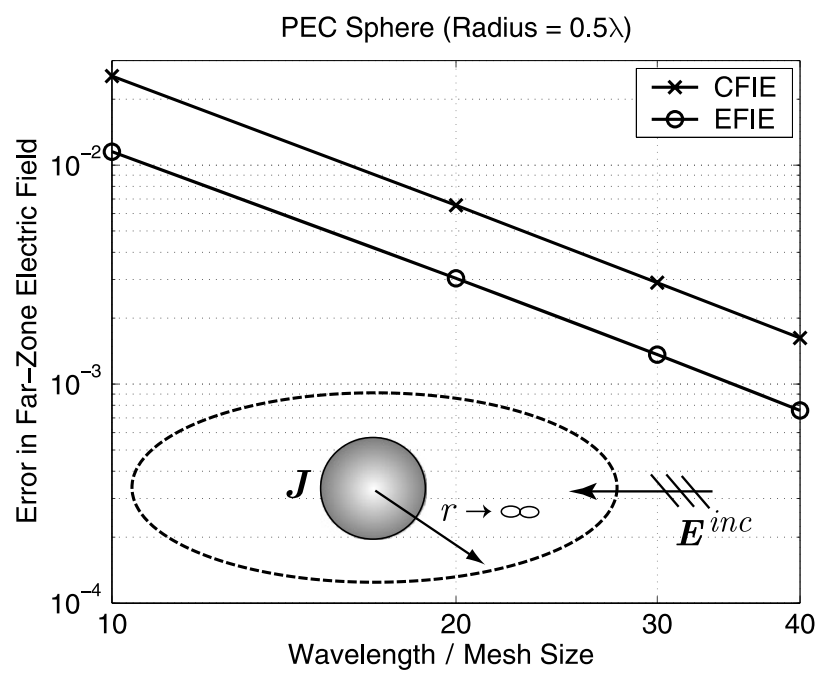

Fig. 2. Error in the electric field scattered from a PEC sphere with a radius of $0.5 \lambda$ illuminated by a plane wave propagating in free space.

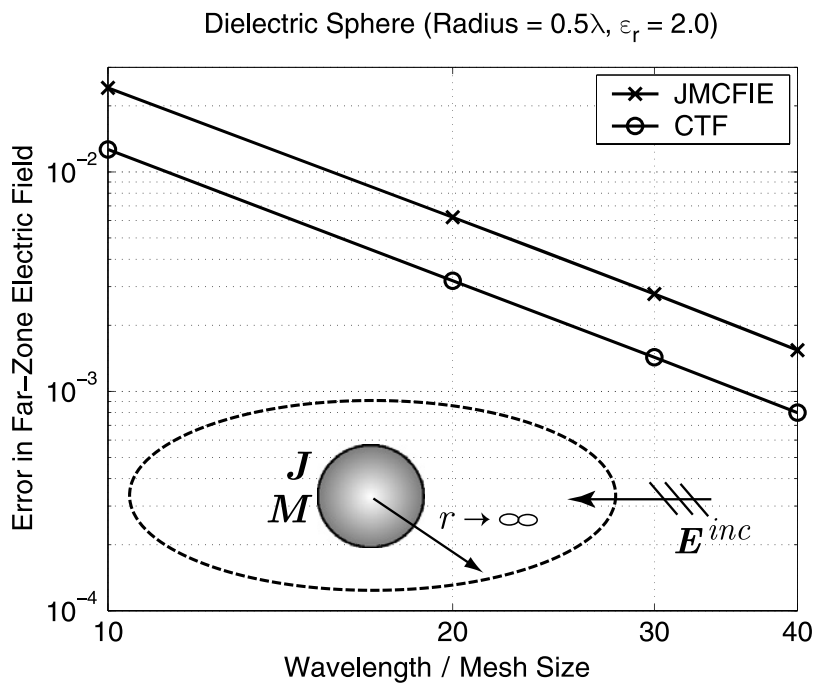

Fig. 3. Error in the electric field scattered from a dielectric sphere with a radius of $0.5 \lambda$ and a relative permittivity of 2.0 and illuminated by a plane wave propagating in free space.

$\phi_{p}=(p-1) \pi / 180$ for $p=1,2, \ldots, 360$. Computational values are compared with the analytical results obtained via a Mie-series solution. A normalized error is defined as

$\Delta=\frac{\operatorname{RMS}\left\{\boldsymbol{E}_{a}^{\infty}-\boldsymbol{E}_{c}^{\infty}\right\}}{\operatorname{RMS}\left\{\boldsymbol{E}_{a}^{\infty}\right\}}$,

where $\boldsymbol{E}_{c}^{\infty}$ and $\boldsymbol{E}_{a}^{\infty}$ are arrays containing the computational and analytical values of the far-zone electric field, respectively. Fig. 2 shows that CFIE, which contains a well-tested identity operator, is significantly more inaccurate than EFIE.

Fig. 3 presents the results of a scattering problem involving a dielectric sphere with a radius of $0.5 \lambda$ and a relative permittivity of 2.0 located in free space. Similar to the previous example, the sphere is illuminated by a plane wave propagating in the $-x$ direction with the electric field polarized in the $y$-direction. The problem is solved with CTF and JMCFIE, and the normalized error in (45) is calculated for various mesh sizes. Fig. 3 shows that CTF is more accurate than JMCFIE, similar to the higher accuracy of EFIE as compared to CFIE.

Finally, we consider the solution of a radiation problem involving a PEC cube with edges of $0.5 \lambda$. A Hertzian dipole oriented in 


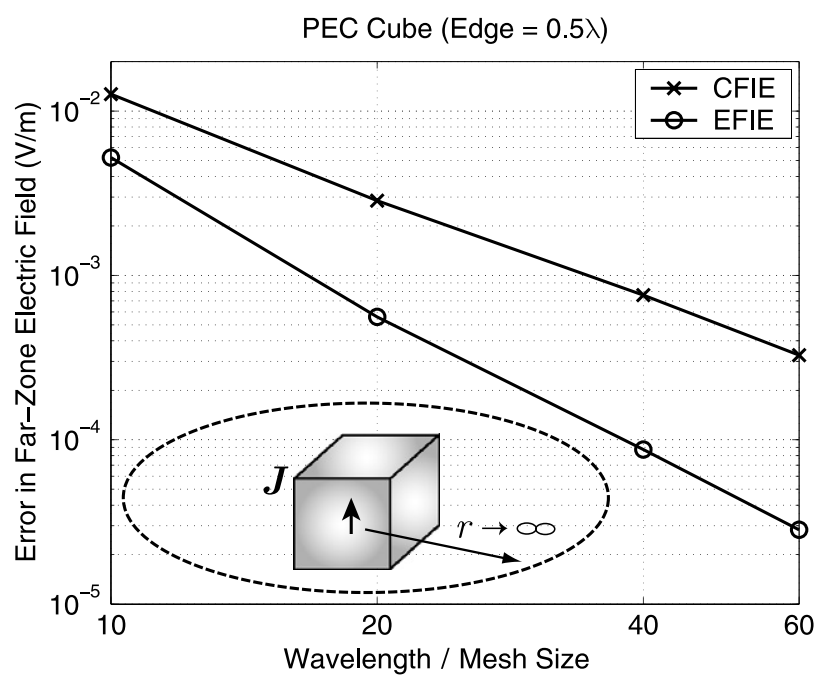

Fig. 4. Error in the electric field radiated by a Hertzian dipole located at the center of a PEC cube with edges of $0.5 \lambda$.

the $z$-direction is located at the center of the cube. Ideally, the radiated field should be zero due to the shielding provided by the conducting cube. Therefore, the nonzero computational fields outside the cube correspond to the error. We calculate the radiated electric field in the far zone on the $x-y$ plane at $\boldsymbol{r}=\left(\infty, \pi / 2, \phi_{p}\right)$, where $\phi_{p}=(p-1) \pi / 180$ for $p=1,2, \ldots, 360$. To normalize the error, we divide the RMS of the total electric field with the RMS of the incident field, i.e.,

$\Delta=\frac{\operatorname{RMS}\left\{\boldsymbol{E}_{\text {tot }}^{\infty}\right\}}{\operatorname{RMS}\left\{\boldsymbol{E}_{\text {inc }}^{\infty}\right\}}$,

where the total electric field is obtained by adding the incident field due to the Hertzian dipole and the secondary field due to the induced electric current on the cube. Fig. 4 shows that the errors in both the EFIE and CFIE solutions decay, as the mesh size is decreased. However, similar to the previous examples, CFIE is significantly less accurate than EFIE.

\section{Concluding remarks}

In this study, we demonstrate the excessive error due to the discretization of the identity operator by using the nonradiating property of the tangential incident fields on arbitrary surfaces. Nonradiating currents $\left\{\boldsymbol{J}^{\text {inc }}(\boldsymbol{r}), \boldsymbol{M}^{\text {inc }}(\boldsymbol{r})\right\}$ are expanded in a series of RWG functions using two different methods. The first method involves the direct testing of the integro-differential $\mathcal{K}$ and $\mathcal{T}$ operators, and it does not contain the identity operator. The second method, however, involves only the identity operator. We show that the second method is significantly inaccurate compared to the first method, even though both of the methods are discretized with the same set of RWG functions. The two methods are analogous to the solutions of electromagnetics problems using first-kind and second-kind integral equations. In fact, solutions with surface integral equations can be interpreted as the expansion of the total currents in a series of basis functions. First-kind equations, such as EFIE, are free of well-tested identity operators, and they are consistently more accurate than the second-kind equations, such as CFIE, involving well-tested identity operators, when these formulations are discretized with low-order basis functions.

\section{Acknowledgements}

This work was supported by the Turkish Academy of Sciences in the framework of the Young Scientist Award Program (LG/TUBAGEBIP/2002-1-12), by the Scientific and Technical Research Council of Turkey (TUBITAK) under Research Grants 105E172 and 107E136, and by contracts from ASELSAN and SSM.

\section{Appendix A. Surface formulations of dielectric problems}

In this appendix, we present the surface formulations of dielectric problems with CTF, MNMF, and JMCFIE. Consider a homogeneous dielectric object with a closed surface separating two media with different electrical properties. Using the equivalence principle, we consider the inner and outer problems separately and derive two sets of equations by employing the boundary conditions, i.e.,

$$
\begin{aligned}
\hat{\boldsymbol{n}} & \times \eta_{o} \mathcal{T}_{0}\{\boldsymbol{J}\}(\boldsymbol{r})-\hat{\boldsymbol{n}} \times \mathcal{K}_{P V, o}\{\boldsymbol{M}\}(\boldsymbol{r})+\frac{\Omega_{0}}{4 \pi} \mathcal{I}\{\boldsymbol{M}\}(\boldsymbol{r}) \\
& =-\hat{\boldsymbol{n}} \times \boldsymbol{E}^{i n c}(\boldsymbol{r}) \quad(\mathrm{NE}-\mathrm{O}), \\
\hat{\boldsymbol{t}} & \cdot \eta_{o} \mathcal{T}_{0}\{\boldsymbol{J}\}(\boldsymbol{r})-\hat{\boldsymbol{t}} \cdot \mathcal{K}_{P V, o}\{\boldsymbol{M}\}(\boldsymbol{r})-\frac{\Omega_{0}}{4 \pi} \hat{\boldsymbol{t}} \cdot \mathcal{I}^{\times n}\{\boldsymbol{M}\}(\boldsymbol{r}) \\
& =-\hat{\boldsymbol{t}} \cdot \boldsymbol{E}^{i n c}(\boldsymbol{r}) \quad(\mathrm{TE}-\mathrm{O}), \\
\hat{\boldsymbol{n}} & \times \frac{1}{\eta_{o}} \mathcal{T}_{0}\{\boldsymbol{M}\}(\boldsymbol{r})+\hat{\boldsymbol{n}} \times \mathcal{K}_{P V, o}\{\boldsymbol{J}\}(\boldsymbol{r})-\frac{\Omega_{0}}{4 \pi} \mathcal{I}\{\boldsymbol{J}\}(\boldsymbol{r}) \\
& =-\hat{\boldsymbol{n}} \times \boldsymbol{H}^{i n c}(\boldsymbol{r}) \quad(\mathrm{NH}-\mathrm{O}), \\
\hat{\boldsymbol{t}} \cdot & \frac{1}{\eta_{0}} \mathcal{T}_{0}\{\boldsymbol{M}\}(\boldsymbol{r})+\hat{\boldsymbol{t}} \cdot \mathcal{K}_{P V, o}\{\boldsymbol{J}\}(\boldsymbol{r})+\frac{\Omega_{0}}{4 \pi} \hat{\boldsymbol{t}} \cdot \mathcal{I}^{\times n}\{\boldsymbol{J}\}(\boldsymbol{r}) \\
& =-\hat{\boldsymbol{t}} \cdot \boldsymbol{H}^{i n c}(\boldsymbol{r}) \quad(\mathrm{TH}-\mathrm{O})
\end{aligned}
$$

for the outer medium denoted with "o" and

$$
\begin{aligned}
& \hat{\boldsymbol{n}} \times \eta_{i} \mathcal{T}_{i}\{\boldsymbol{J}\}(\boldsymbol{r})-\hat{\boldsymbol{n}} \times \mathcal{K}_{P V, i}\{\boldsymbol{M}\}(\boldsymbol{r})-\frac{\Omega_{i}}{4 \pi} \mathcal{I}\{\boldsymbol{M}\}(\boldsymbol{r})=0 \quad(\mathrm{NE}-\mathrm{I}), \\
& \hat{\boldsymbol{t}} \cdot \eta_{i} \mathcal{T}_{i}\{\boldsymbol{J}\}(\boldsymbol{r})-\hat{\boldsymbol{t}} \cdot \mathcal{K}_{P V, i}\{\boldsymbol{M}\}(\boldsymbol{r})+\frac{\Omega_{i}}{4 \pi} \hat{\boldsymbol{t}} \cdot \mathcal{I}^{\times n}\{\boldsymbol{M}\}(\boldsymbol{r})=0 \quad(\mathrm{TE}-\mathrm{I}),
\end{aligned}
$$

$\hat{\boldsymbol{n}} \times \frac{1}{\eta_{i}} \mathcal{T}_{i}\{\boldsymbol{M}\}(\boldsymbol{r})+\hat{\boldsymbol{n}} \times \mathcal{K}_{P V, i}\{\boldsymbol{J}\}(\boldsymbol{r})+\frac{\Omega_{i}}{4 \pi} \mathcal{I}\{\boldsymbol{J}\}(\boldsymbol{r})=0 \quad(\mathrm{NH}-\mathrm{I})$,

$\hat{\boldsymbol{t}} \cdot \frac{1}{\eta_{i}} \mathcal{T}_{i}\{\boldsymbol{M}\}(\boldsymbol{r})+\hat{\boldsymbol{t}} \cdot \mathcal{K}_{P V, i}\{\boldsymbol{J}\}(\boldsymbol{r})-\frac{\Omega_{i}}{4 \pi} \hat{\boldsymbol{t}} \cdot \mathcal{I}^{\times n}\{\boldsymbol{J}\}(\boldsymbol{r})=0$

for the inner medium denoted with " $i$ ". CTF is obtained by combining the tangential equations as [3]

$\left[\begin{array}{c}\eta_{o}^{-1} \mathrm{TE}-\mathrm{O}+\eta_{i}^{-1} \mathrm{TE}-\mathrm{I} \\ \eta_{0} \mathrm{TH}-\mathrm{O}+\eta_{i} \mathrm{TH}-\mathrm{I}\end{array}\right]$

leading to

$\hat{\boldsymbol{t}} \cdot\left[\begin{array}{c}Z_{11}^{T}(\boldsymbol{r})+Z_{12}^{T}(\boldsymbol{r}) \\ Z_{21}^{T}(\boldsymbol{r})+Z_{22}^{T}(\boldsymbol{r})\end{array}\right]=-\hat{\boldsymbol{t}} \cdot\left[\begin{array}{c}\eta_{o}^{-1} \boldsymbol{E}^{i n c}(\boldsymbol{r}) \\ \eta_{o} \boldsymbol{H}^{\text {inc }}(\boldsymbol{r})\end{array}\right]$,

where

$$
\begin{aligned}
Z_{11}^{T}(\boldsymbol{r})= & \left(\mathcal{T}_{0}+\mathcal{T}_{i}\right)\{\boldsymbol{J}\}(\boldsymbol{r}), \\
Z_{12}^{T}(\boldsymbol{r})= & -\left(\eta_{o}^{-1} \mathcal{K}_{P V, o}+\eta_{i}^{-1} \mathcal{K}_{P V, i}\right)\{\boldsymbol{M}\}(\boldsymbol{r}) \\
& -\frac{1}{4 \pi}\left(\Omega_{o} \eta_{o}^{-1}-\Omega_{i} \eta_{i}^{-1}\right) \mathcal{I}^{\times n}\{\boldsymbol{M}\}(\boldsymbol{r}), \\
Z_{21}^{T}(\boldsymbol{r})= & \left(\eta_{o} \mathcal{K}_{P V, o}+\eta_{i} \mathcal{K}_{P V, i}\right)\{\boldsymbol{J}\}(\boldsymbol{r}) \\
& +\frac{1}{4 \pi}\left(\Omega_{0} \eta_{o}-\Omega_{i} \eta_{i}\right) \mathcal{I}^{\times n}\{\boldsymbol{J}\}(\boldsymbol{r}), \\
Z_{22}^{T}(\boldsymbol{r})= & \left(\mathcal{T}_{0}+\mathcal{T}_{i}\right)\{\boldsymbol{M}\}(\boldsymbol{r}) .
\end{aligned}
$$

Using a Galerkin scheme, the discretization of CTF involves weaklytested identity operators, i.e.,

$\int_{S_{m}} d \boldsymbol{r} \boldsymbol{t}_{m}(\boldsymbol{r}) \cdot \mathcal{I}^{\times n}\left\{\boldsymbol{b}_{n}\right\}(\boldsymbol{r})=\int_{S_{m}} d \boldsymbol{r} \boldsymbol{t}_{m}(\boldsymbol{r}) \cdot \hat{\boldsymbol{n}} \times \boldsymbol{b}_{n}(\boldsymbol{r})$. 
Unlike CTF, MNMF is obtained by combining the normal equations as [4]

$\left[\begin{array}{c}\mu_{o} /\left(\mu_{o}+\mu_{i}\right) \mathrm{NH}-\mathrm{O}-\mu_{i} /\left(\mu_{o}+\mu_{i}\right) \mathrm{NH}-\mathrm{I} \\ -\epsilon_{o} /\left(\epsilon_{o}+\epsilon_{i}\right) \mathrm{NE}-\mathrm{O}+\epsilon_{i} /\left(\epsilon_{o}+\epsilon_{i}\right) \mathrm{NE}-\mathrm{I}\end{array}\right]$,

leading to

$\hat{\boldsymbol{n}} \times\left[\begin{array}{c}Z_{11}^{N}(\boldsymbol{r})+Z_{12}^{N}(\boldsymbol{r}) \\ Z_{21}^{N}(\boldsymbol{r})+Z_{22}^{N}(\boldsymbol{r})\end{array}\right]=-\hat{\boldsymbol{n}} \times\left[\begin{array}{c}\mu_{0} \boldsymbol{H}^{i n c}(\boldsymbol{r}) /\left(\mu_{0}+\mu_{i}\right) \\ -\epsilon_{0} \boldsymbol{E}^{i n c}(\boldsymbol{r}) /\left(\epsilon_{0}+\epsilon_{i}\right)\end{array}\right]$,

where

$$
\begin{aligned}
Z_{11}^{N}(\boldsymbol{r})= & \frac{1}{\left(\mu_{o}+\mu_{i}\right)}\left(\mu_{o} \mathcal{K}_{P V, o}-\mu_{i} \mathcal{K}_{P V, i}\right)\{\boldsymbol{J}\}(\boldsymbol{r}) \\
& +\frac{1}{4 \pi\left(\mu_{0}+\mu_{i}\right)}\left(\mu_{0} \Omega_{o}+\mu_{i} \Omega_{i}\right) \mathcal{I}^{\times n}\{\boldsymbol{J}\}(\boldsymbol{r}), \\
Z_{12}^{N}(\boldsymbol{r})= & \frac{1}{\left(\mu_{o}+\mu_{i}\right)}\left(\mu_{o} \eta_{o}^{-1} \mathcal{T}_{o}-\mu_{i} \eta_{i}^{-1} \mathcal{T}_{i}\right)\{\boldsymbol{M}\}(\boldsymbol{r}), \\
Z_{21}^{N}(\boldsymbol{r})= & \frac{1}{\left(\epsilon_{0}+\epsilon_{i}\right)}\left(\epsilon_{i} \eta_{i} \mathcal{T}_{i}-\epsilon_{o} \eta_{o} \mathcal{T}_{0}\right)\{\boldsymbol{J}\}(\boldsymbol{r}), \\
Z_{22}^{N}(\boldsymbol{r})= & \frac{1}{\left(\epsilon_{0}+\epsilon_{i}\right)}\left(\epsilon_{o} \mathcal{K}_{P V, o}-\epsilon_{i} \mathcal{K}_{P V, i}\right)\{\boldsymbol{M}\}(\boldsymbol{r}) \\
& +\frac{1}{4 \pi\left(\epsilon_{o}+\epsilon_{i}\right)}\left(\epsilon_{o} \Omega_{o}+\epsilon_{i} \Omega_{i}\right) \mathcal{I}^{\times n}\{\boldsymbol{M}\}(\boldsymbol{r}) .
\end{aligned}
$$

MNMF is a second-kind integral equation, and its discretization involves well-tested identity operators, i.e.,

$\int_{S_{m}} d \boldsymbol{r} \boldsymbol{t}_{m}(\boldsymbol{r}) \cdot \hat{\boldsymbol{n}} \times \mathcal{I}^{\times n}\left\{\boldsymbol{b}_{n}\right\}(\boldsymbol{r})=-\int_{S_{m}} d \boldsymbol{r} \boldsymbol{t}_{m}(\boldsymbol{r}) \cdot \boldsymbol{b}_{n}(\boldsymbol{r})$,

using a Galerkin scheme.

CTF and MNMF are natural extensions of EFIE and MFIE, respectively, for the solution of dielectric problems. For PEC objects, CTF in (56) reduces to two decoupled equations, i.e.,

$$
\begin{aligned}
& \hat{\boldsymbol{t}} \cdot \eta_{o} \mathcal{T}_{0}\{\boldsymbol{J}\}(\boldsymbol{r})=-\hat{\boldsymbol{t}} \cdot \boldsymbol{E}^{i n c}(\boldsymbol{r}), \\
& \hat{\boldsymbol{t}} \cdot \mathcal{K}_{P V, 0}\{\boldsymbol{J}\}(\boldsymbol{r})+\hat{\boldsymbol{t}} \cdot \frac{\Omega_{0}}{4 \pi} \mathcal{I}^{\times n}\{\boldsymbol{J}\}(\boldsymbol{r})=-\hat{\boldsymbol{t}} \cdot \boldsymbol{H}^{i n c}(\boldsymbol{r}) .
\end{aligned}
$$

The first equation (69) is the original EFIE, whereas the second equation (70) is a tangential form of MFIE, which is unstable when it is discretized by using a Galerkin scheme, since both $\mathcal{K}$ and identity operators are weakly tested. Similarly, for PEC objects, MNMF in (63) reduces to two decoupled equations. These are the original MFIE

$\hat{\boldsymbol{n}} \times \mathcal{K}_{P V, o}\{\boldsymbol{J}\}(\boldsymbol{r})-\frac{\Omega_{0}(\boldsymbol{r})}{4 \pi} \mathcal{I}\{\boldsymbol{J}\}(\boldsymbol{r})=-\hat{\boldsymbol{n}} \times \boldsymbol{H}^{i n c}(\boldsymbol{r})$

and a normal form of EFIE, i.e.,

$\hat{\boldsymbol{n}} \times \eta_{0} \mathcal{T}_{0}\{\boldsymbol{J}\}(\boldsymbol{r})=-\hat{\boldsymbol{n}} \times \boldsymbol{E}^{i n c}(\boldsymbol{r})$,

which is unstable when it is discretized with a Galerkin scheme, since the $\mathcal{T}$ operator is weakly tested.

Finally, as an extension of CFIE for dielectric objects, JMCFIE can be derived by combining all equations in (47)-(54) as [5]

$\left[\begin{array}{c}\eta_{o}^{-1} \mathrm{TE}-\mathrm{O}+\eta_{i}^{-1} \mathrm{TE}-\mathrm{I}+\mathrm{NH}-\mathrm{O}-\mathrm{NH}-\mathrm{I} \\ \eta_{o} \mathrm{TH}-\mathrm{O}+\eta_{i} \mathrm{TH}-\mathrm{I}-\mathrm{NE}-\mathrm{O}+\mathrm{NE}-\mathrm{I}\end{array}\right]$
For PEC objects, JMCFIE reduces to two decoupled equations: the original CFIE and an unstable form of CFIE.

\section{References}

[1] A.J. Poggio, E.K. Miller, Integral equation solutions of three-dimensional scattering problems, in: R. Mittra (Ed.), Computer Techniques for Electromagnetics, Pergamon Press, Oxford, 1973 (Ch. 4).

[2] J.R. Mautz, R.F. Harrington, H-field, E-field, and combined field solutions for conducting bodies of revolution, AEÜ 32 (4) (1978) 157-164.

[3] P. Ylä-Oijala, M. Taskinen, S. Järvenpää, Surface integral equation formulations for solving electromagnetic scattering problems with iterative methods, Radio Sci. 40 (2005) RS6002, doi:10.1029/2004RS003169.

[4] P. Ylä-Oijala, M. Taskinen, Well-conditioned Müller formulation for electromagnetic scattering by dielectric objects, IEEE Trans. Antennas Propag. 53 (10) (2005) 3316-3323.

[5] P. Ylä-Oijala, M. Taskinen, Application of combined field integral equation for electromagnetic scattering by dielectric and composite objects, IEEE Trans. Antennas Propag. 53 (3) (2005) 1168-1173.

[6] C. Müller, Foundations of the Mathematical Theory of Electromagnetic Waves, Springer, New York, 1969.

[7] Ö. Ergül, L. Gürel, Efficient parallelization of the multilevel fast multipole algorithm for the solution of large-scale scattering problems, IEEE Trans. Antennas Propag. 56 (8) (2008) 2335-2345.

[8] Ö. Ergül, L. Gürel, Investigation of the inaccuracy of the MFIE discretized with the RWG basis functions, in: Proc. IEEE Antennas and Propagation Soc. Int. Symp., vol. 3, 2004, pp. 3393-3396.

[9] C.P. Davis, K.F. Warnick, High-order convergence with a low-order discretization of the 2-D MFIE, IEEE Antennas Wireless Propag. Lett. 3 (2004) 355-358.

[10] S.M. Rao, D.R. Wilton, A.W. Glisson, Electromagnetic scattering by surfaces of arbitrary shape, IEEE Trans. Antennas Propag. AP-30 (3) (May 1982) 409418.

[11] Ö. Ergül, L. Gürel, Improving the accuracy of the MFIE with the choice of basis functions, in: Proc. IEEE Antennas and Propagation Soc. Int. Symp., vol. 3, 2004, pp. 3389-3392.

[12] E. Ubeda, J.M. Rius, MFIE MOM-formulation with curl-conforming basis functions and accurate kernel integration in the analysis of perfectly conducting sharp-edged objects, Microw. Opt. Technol. Lett. 44 (4) (2005) 354-358.

[13] E. Ubeda, J.M. Rius, Monopolar divergence-conforming and curl-conforming low-order basis functions for the electromagnetic scattering analysis, Microw. Opt. Technol. Lett. 46 (3) (2005) 237-241.

[14] E. Ubeda, J.M. Rius, Novel monopolar MFIE MoM-discretization for the scattering analysis of small objects, IEEE Trans. Antennas Propag. 54 (1) (2006) 50-57.

[15] Ö. Ergül, L. Gürel, The use of curl-conforming basis functions for the magneticfield integral equation, IEEE Trans. Antennas Propag. 54 (2006) 1917-1926.

[16] Ö. Ergül, L. Gürel, Improving the accuracy of the magnetic field integral equation with the linear-linear basis functions, Radio Sci. 41 (2006) RS4004, doi:10.1029/2005RS003307.

[17] Ö. Ergül, L. Gürel, Linear-linear basis functions for MLFMA solutions of magnetic-field and combined-field integral equations, IEEE Trans. Antennas Propag. 55 (4) (2007) 1103-1110.

[18] K.F. Warnick, A.F. Peterson, 3D MFIE accuracy improvement using regularization, in: Proc. IEEE Antennas and Propagation Soc. Int. Symp., 2007, pp. 48574860.

[19] N. Morita, N. Kumagai, J.R. Mautz, Integral Equation Methods for Electromagnetics, Artech House, Boston, 1990.

[20] L. Gürel, Ö. Ergül, Comparisons of FMM implementations employing different formulations and iterative solvers, in: Proc. IEEE Antennas and Propagation Soc. Int. Symp., vol. 1, 2003, pp. 19-22.

[21] G.C. Hsiao, R.E. Kleinman, Mathematical foundations for error estimation in numerical solutions of integral equations in electromagnetics, IEEE Trans. Antennas Propag. 45 (3) (1997) 316-328.

[22] Ö. Ergül, L. Gürel, Stabilization of integral-equation formulations for the accurate solution of scattering problems involving low-contrast dielectric objects, IEEE Trans. Antennas Propag. 56 (3) (2008) 799-805.

[23] P. Ylä-Oijala, M. Taskinen, S. Järvenpää, Analysis of surface integral equations in electromagnetic scattering and radiation problems, Eng. Anal. Boundary Elem. 32 (3) (2008) 196-209. 